Research Article

\title{
Nonlinear Dynamics and Suppressing Chaos in Magnetic Bearing System
}

\author{
Shun-Chang Chang (i) \\ Department of Mechanical and Automation Engineering, Da-Yeh University, Changhua 51591, Taiwan \\ Correspondence should be addressed to Shun-Chang Chang; changsc@mail.dyu.edu.tw
}

Received 4 November 2020; Revised 5 December 2020; Accepted 12 December 2020; Published 23 December 2020

Academic Editor: A. M. Abd-Alla

Copyright (c) 2020 Shun-Chang Chang. This is an open access article distributed under the Creative Commons Attribution License, which permits unrestricted use, distribution, and reproduction in any medium, provided the original work is properly cited.

\begin{abstract}
A nonlinear mathematical model of a magnetic bearing system has been obtained by applying a modified conventional identification technique based on the principle of harmonic balance. In this study, we examined the rich nonlinear dynamics of a magnetic bearing system with closed-loop control using phase portraits, Poincaré maps, and frequency spectra. The resulting bifurcation diagram can be used to evaluate the operational range of systems employing nonlinear actuators. Estimates of the largest Lyapunov exponent based on the properties of synchronization revealed the occurrence of chatter vibration indicative of chaotic motion. Various control methods, such as the state feedback control and the injection of dither signals, were then used to quench the chaotic behavior.
\end{abstract}

\section{Introduction and System Description}

The rotor in magnetic bearing systems is suspended by magnetic bearings to ensure stable rotation at high speeds; however, a closed-loop control system is required to stabilize the system by eliminating vibrations caused by disturbing forces. The characteristics of magnetic bearings are inherently nonlinear due to nonlinearities in electromagnetic forces. The occurrence of large unbalanced forces in rotor bearings can cause nonlinear motion of high amplitude in magnetic bearing systems. Accurate control of the system is required which designers account for the effects of nonlinearities. In a previous work [1], we conducted experiments on an unloaded symmetric rotor with flexible coupler at one end and a bearing comprising two pairs of electromagnets at the other end (Figure 1). It was carried out by applying a series of nonlinear electromagnetic forces to identify a nonlinear model for this system. These rich nonlinear dynamics must be taken into account in the design of magnetic bearing systems. In this study, we sought to predict these nonlinear dynamics by modifying the conventional identification technique based on the principle of harmonic balance in order to characterize the system with a higher degree of precision. The resulting nonlinear model [1] is obtained as follows:

$$
\begin{aligned}
& \dot{x}_{1}=x_{2} \text {, } \\
& \dot{x}_{2}=-d_{1} x_{2}-d_{2} x_{1}+d_{3} x_{3}+d_{4} x_{4}+d_{5} x_{1}^{2}+d_{6} x_{1} x_{3} \\
& +d_{7} x_{1} x_{4}+d_{8} x_{3}^{2}+d_{9} x_{4}^{2}+d_{10} x_{1}^{3} \text {, } \\
& L \dot{x}_{3}+R x_{3}=K_{A}\left(e_{c 1}+A_{0} \sin \Omega t\right), \\
& L \dot{x}_{4}+R x_{4}=K_{A}\left(e_{c 2}+A_{0} \sin \Omega t\right),
\end{aligned}
$$

where

$$
\begin{aligned}
& e_{c 1}=K_{H}\left(K_{P} x_{1}+K_{D} x_{2}+K_{I} \int x_{1} \mathrm{~d} t\right), \\
& e_{c 2}=K_{H}\left(-K_{P} x_{1}-K_{D} x_{2}-K_{I} \int x_{1} \mathrm{~d} t\right),
\end{aligned}
$$




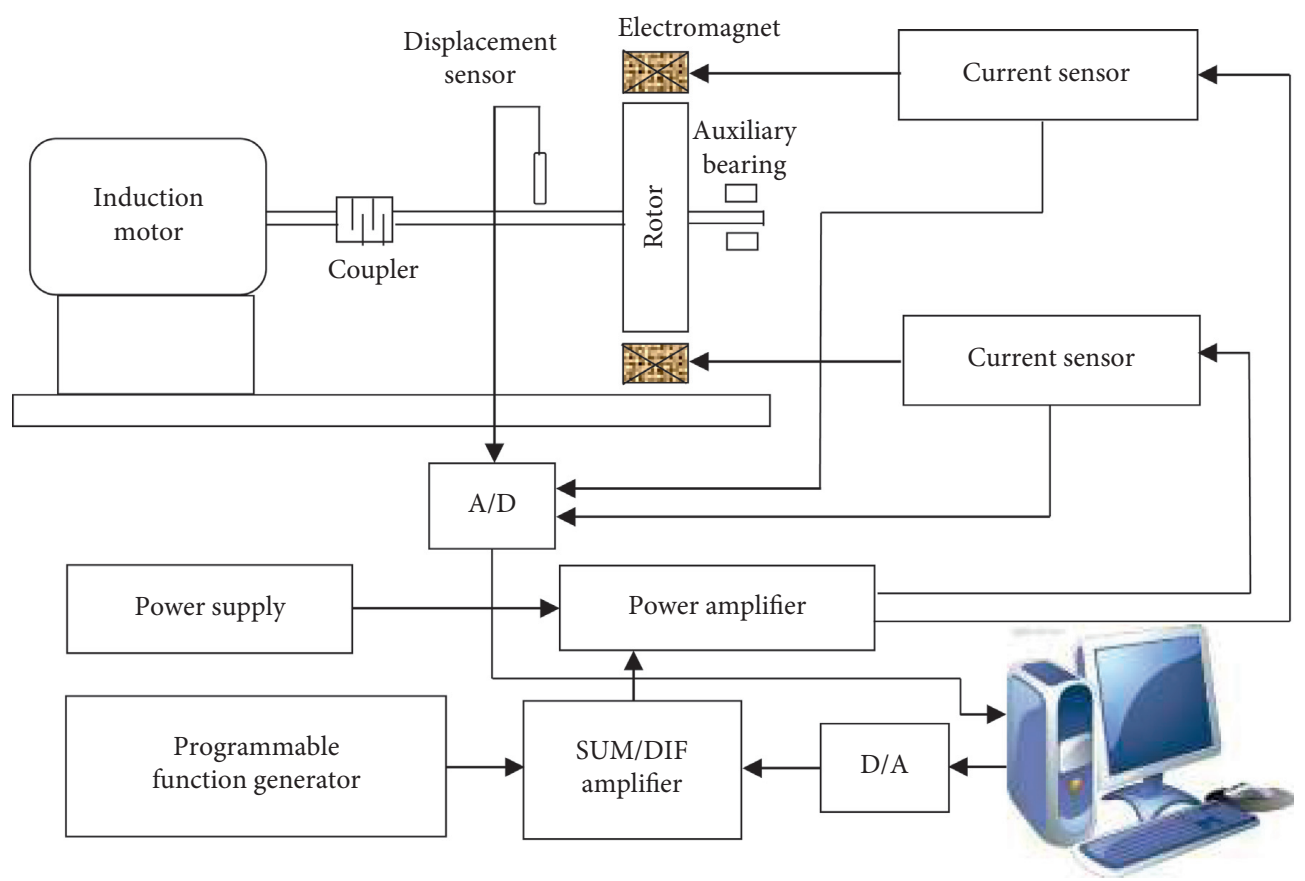

FIgURE 1: Schematic diagram of a magnetic bearing system.

with the limitations

$$
\begin{array}{r}
-3.5 \text { volts }<e_{c 1}<6.5 \text { volts, } \\
-3.5 \text { volts }<e_{c 2}<6.5 \text { volts, } \\
0.0 \text { amp }<x_{3} \text { and } x_{4}<2.0 \text { amp, }
\end{array}
$$

where $x_{1}$ is the displacement of the rotor around the equilibrium point; $x_{2}$ is the velocity of the rotor; $x_{3}$ and $x_{4}$ are coil currents oscillating around the bias current; $e_{c 1}$ and $e_{c 2}$ are the outputs of the PID controller to the two coils; $K_{P}=-55, K_{D}=-0.3$, and $K_{I}=-50$ are the control gains; $K_{A}$ $(=2.4)$ and $K_{H}(=10000)$ represent the gains of the power amplifier and displacement sensor; and $A_{0} \sin \Omega t$ is the forcing voltage generated by a programmable function generator. The procedures used to derive the other coefficients necessary for (1a)-(1d) are listed in Table 1 [1].

This model captures the primary characteristics of the system by comparing the frequency responses from simulations with those from experiments [1]. However, this model has not been subjected to dynamic analysis to determine whether the nonlinear mathematical model derived from experiments can be used to characterize and/ or predict the dynamics in a physical system. Furthermore, the chaotic motion that occurs at the moment the rotor strikes the electromagnet has not been characterized.

In this study, we employed bifurcation diagrams, phase portraits, Poincaré maps, frequency spectra, and Lyapunov exponents to observe periodic and chaotic motions. Across a broad range of parameters, the Lyapunov exponent provides the most powerful means by which to measure the sensitivity of a dynamic system as it pertains to its initial conditions. This approach can be used to determine whether a system is susceptible to chaotic motion. The algorithms used to
TABLE 1: Identified results [1].

\begin{tabular}{lc}
\hline System parameter & Identified value \\
\hline$d_{1}$ & 3.066133 \\
$d_{2}$ & $4.3315 \times 10^{3}$ \\
$d_{3}$ & -5.5786683 \\
$d_{4}$ & 6.5562 \\
$d_{5}$ & $1.43371 \times 10^{7}$ \\
$d_{6}$ & $-2.531 \times 10^{4}$ \\
$d_{7}$ & $-4.53203 \times 10^{4}$ \\
$d_{8}$ & 0.34514 \\
$d_{9}$ & -0.40171 \\
$d_{10}$ & $-2.399816 \times 10^{10}$ \\
$L$ & 0.0161203 \\
$R$ & 14.3128231 \\
\hline
\end{tabular}

compute Lyapunov exponents associated with smooth dynamic systems are well-established [2-5]. However, a number of nonsmooth dynamic systems possess discontinuities to which this algorithm cannot be applied directly, such as those associated with dry friction, backlash, and saturation. Many studies have proposed methods for the calculation of Lyapunov exponents associated with nonsmooth dynamic systems [6-8]. In this study, we adopted the method developed by Stefanski [8] for estimating the largest Lyapunov exponent in a magnetic bearing system with closed-loop control.

Many practical engineering problems involving chaos require control techniques to convert chaotic attractors into stable periodic orbits. Since the pioneering work of Ott et al. [9], numerous methods for the control of chaos have been devised [10-19]. Improving the performance of a controlled magnetic bearing system and/or eliminating chatter behavior require the conversion of chaotic behavior into periodic motion. Two control methods have been developed 
for chaos suppression: state feedback control [11-15] and dither control [16-19].

\section{System Characteristics: Simulations and Discussion}

We conducted a series of numerical simulations based on (1a)-(1d) to clearly elucidate the dynamic characteristics of the system in this study. In (1a)-(1d), the amplitude of the input excitation, $A_{0}$, equals $3.5 \mathrm{~V}$. We employed FORTRAN subroutines in the commercial software package DIVPRK (IMSL) to solve ordinary differential equations [20]. The resulting bifurcation diagram is presented in Figure 2. This figure clearly shows that the first period-doubling bifurcation occurred at approximately $\Omega=19.5 \mathrm{~Hz}$ and chaotic motion appeared at approximately $\Omega=18.5 \mathrm{~Hz}$. Further details of the responses exhibited by the system are presented in Figures $3-5$, in which each type of response is characterized by a phase portrait, Poincaré map, and frequency spectrum. Figures 3(a)-3(c) show that the $T_{f}$-period includes the constant term and fundamental components. From Figure 4, we determined that a cascade of period-doubling bifurcations produced a series of subharmonic components, revealing the bifurcations with new frequency components at $\Omega / 2,3 \Omega / 2$, $5 \Omega / 2, \ldots$. The essence of chaotic behavior can be described using Poincaré maps, which present an infinite set of points referred to as a strange attractor. Chaotic motion also presents a broad continuous frequency spectrum. Thus, strange attractors and continuous-type Fourier spectra are generally regarded as strong indictors of chaos, as illustrated in Figures 5(a)-5(c).

\section{Synchronization and the Lyapunov Exponent}

The largest Lyapunov exponent is a useful diagnostic element for the analysis of chaotic systems. Every dynamic system possesses a spectrum of Lyapunov exponents $(\lambda)$, which determine length, area, and volume changes in the phase space. In other words, Lyapunov exponents measure the rate of divergence (or convergence) between two adjacent orbits. Chaos can be identified simply by calculating the largest Lyapunov exponent, thereby determining whether nearby trajectories generally diverge $(\lambda>0)$ or converge $(\lambda<0)$. Any bounded motion in a system containing at least one positive Lyapunov exponent is defined as chaotic, whereas nonpositive Lyapunov exponents indicate periodic motion. There are a number of well-established algorithms that compute the Lyapunov spectrum of smooth dynamic systems [2-5]. However, nonsmooth dynamic systems with discontinuities, such as dry friction, backlash, and saturation, do not allow for the direct application of such algorithms. In this study, we estimated the largest Lyapunov exponent in order to identify the onset of chaotic motion in a controlled magnetic bearing system. Stefanski [8] proposed a simple method for estimating the largest Lyapunov exponent based on properties associated with synchronization. Synchronization controls the response system by accessing the output of the drive system such that the output of the

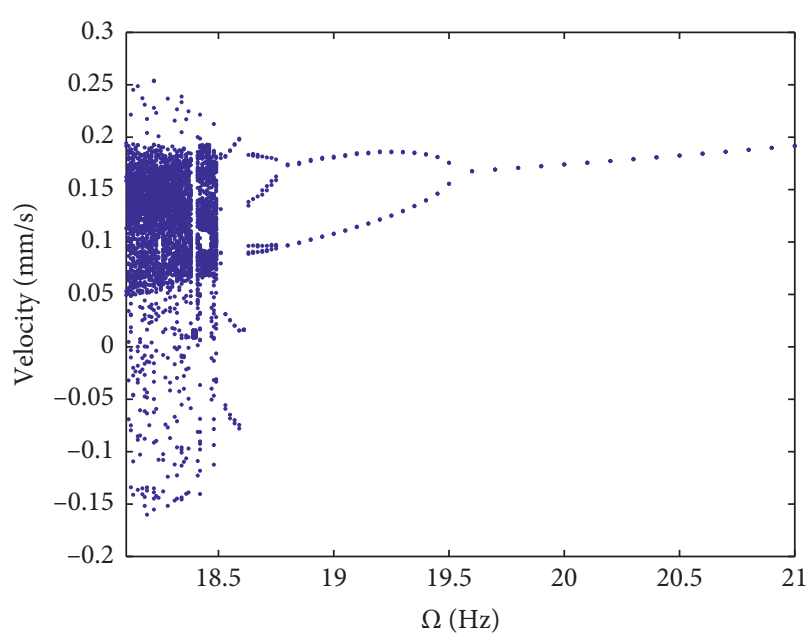

Figure 2: Bifurcation diagram of the system for $A_{0}=3.5 \mathrm{~V}$.

response system asymptotically follows the output of the drive system. This method is described briefly as follows.

The dynamic system is decomposed into the following two subsystems:

A drive system

$$
\dot{x}=f(x) .
$$

A response system

$$
\dot{y}=f(y) .
$$

Consider a dynamic system comprising two identical $n$-dimensional subsystems, where the response system (5) is combined with coupling coefficient $d$, and the drive system (4) remains the same. The first-order differential equations used to describe such a system are written as follows:

$$
\begin{aligned}
& \dot{x}=f(x), \\
& \dot{y}=f(y)+d(x-y) .
\end{aligned}
$$

The condition of synchronization is given by the following inequality:

$$
d>\lambda_{\max }
$$

The smallest value of coupling coefficient $d$ in synchronization $d_{s}$ is assumed to be equal to the largest Lyapunov exponent, as follows:

$$
d_{s}=\lambda_{\max }
$$

(6) provides an augmented system based on (1a)-(1d), as follows:

$$
\begin{aligned}
\dot{x}_{1}= & x_{2}, \\
\dot{x}_{2}= & -d_{1} x_{2}-d_{2} x_{1}+d_{3} x_{3}+d_{4} x_{4}+d_{5} x_{1}^{2}+d_{6} x_{1} x_{3} \\
& +d_{7} x_{1} x_{4}+d_{8} x_{3}^{2}+d_{9} x_{4}^{2}+d_{10} x_{1}^{3},
\end{aligned}
$$




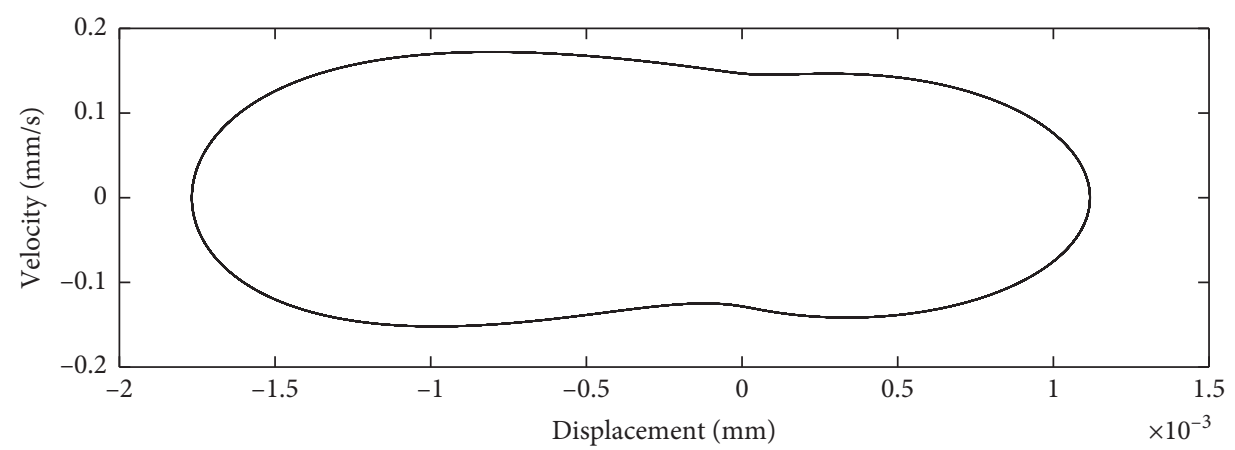

(a)

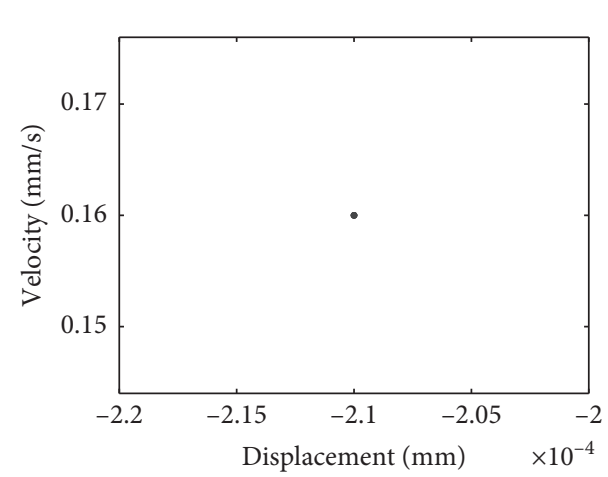

(b)

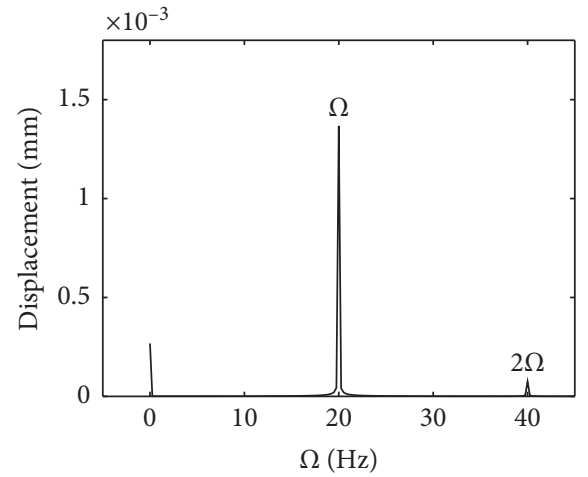

(c)

Figure 3: Period-one orbit for $\Omega=20.0 \mathrm{~Hz}$ : (a) phase portrait; (b) Poincaré map; (c) frequency spectrum.

$$
\begin{gathered}
L \dot{x}_{3}+R x_{3}=K_{A}\left(e_{c 1 x}+A_{0} \sin \Omega t\right) \\
L \dot{x}_{4}+R x_{4}=K_{A}\left(e_{c 2 x}+A_{0} \sin \Omega t\right) \\
\dot{y}_{1}=y_{2}+d\left(x_{1}-y_{1}\right) \\
\dot{y}_{2}=-d_{1} y_{2}-d_{2} y_{1}+d_{3} y_{3}+d_{4} y_{4}+d_{5} y_{1}^{2}+d_{6} y_{1} y_{3} \\
+d_{7} y_{1} y_{4}+d_{8} y_{3}^{2}+d_{9} y_{4}^{2}+d_{10} y_{1}^{3}+d\left(x_{2}-y_{2}\right) \\
L \dot{y}_{3}+R y_{3}=K_{A}\left(e_{c 1 y}+A_{0} \sin \Omega t\right)+d\left(x_{3}-y_{3}\right) \\
L \dot{y}_{4}+R y_{4}=K_{A}\left(e_{c 2 y}+A_{0} \sin \Omega t\right)+d\left(x_{4}-y_{4}\right)
\end{gathered}
$$

where

$$
\begin{aligned}
& e_{c 1 x}=K_{H}\left(K_{P} x_{1}+K_{D} x_{2}+K_{I} \int x_{1} \mathrm{~d} t\right), \\
& e_{c 2 x}=K_{H}\left(-K_{P} x_{1}-K_{D} x_{2}-K_{I} \int x_{1} \mathrm{~d} t\right), \\
& e_{c 1 y}=K_{H}\left(K_{P} y_{1}+K_{D} y_{2}+K_{I} \int y_{1} \mathrm{~d} t\right), \\
& e_{c 2 y}=K_{H}\left(-K_{P} y_{1}-K_{D} y_{2}-K_{I} \int y_{1} \mathrm{~d} t\right),
\end{aligned}
$$

with the following limitations:

$$
\begin{array}{r}
-3.5 \text { volts }<e_{c 1 x}<6.5 \text { volts, } \\
-3.5 \text { volts }<e_{c 2 x}<6.5 \text { volts, } \\
0.0 \text { amp }<x_{3} \text { and } x_{4}<2.0 \text { amp, } \\
-3.5 \text { volts }<e_{c 1 y}<6.5 \text { volts, } \\
-3.5 \text { volts }<e_{c 2 y}<6.5 \text { volts, } \\
0.0 \text { amp }<y_{3} \text { and } y_{4}<2.0 \text { amp. }
\end{array}
$$

In the next step, we estimate the largest Lyapunov exponent for the selected parametric values in accordance with the method described above. Figure 6 presents the results of numerical calculations showing the estimated largest Lyapunov exponents obtained using the above synchronization method. All of the largest Lyapunov exponents are positive with regard to the forcing frequency $(\Omega<18.5 \mathrm{~Hz})$, indicating that the system exhibits chaotic motion. These results help to enhance our understanding of chatter vibration in a controlled magnetic bearing system.

\section{Quenching Chatter}

Chaotic motion can induce chatter vibrations capable of destroying a magnetic bearing system. Accurate prediction of the behavior of a chaotic system can be beneficial; 


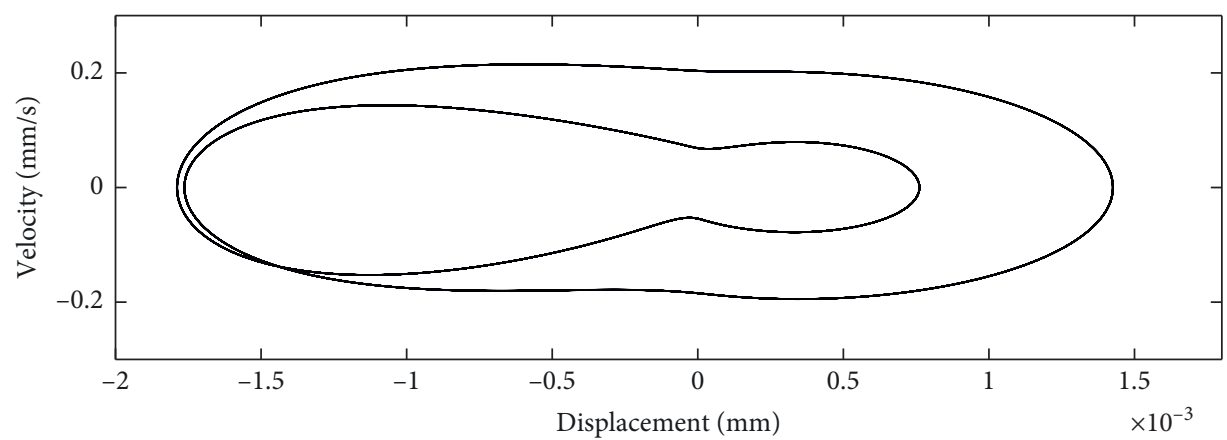

(a)

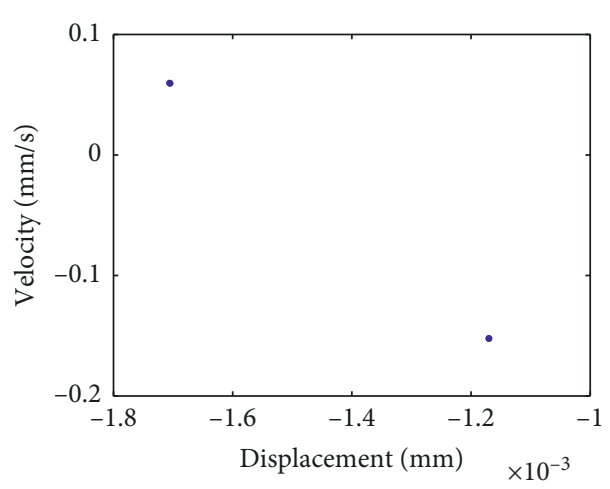

(b)

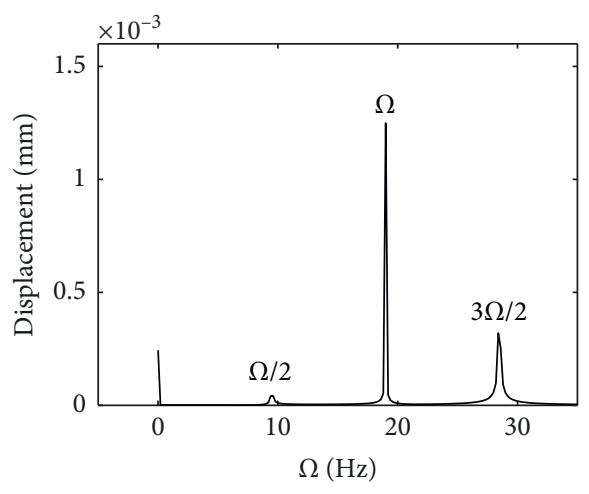

(c)

FIgure 4: Period-two orbit for $\Omega=19.0 \mathrm{~Hz}$ : (a) phase portrait; (b) Poincaré map; (c) frequency spectrum.

however, maximizing the benefits requires the ability to control this behavior. A chaotic system must be transformed into periodic motion in order to work under specific conditions and thereby improve the performance of a dynamic system and/or avoid chatter. In this section, we discuss the means by which chaos can be converted into periodic motion using minimal efforts. We present two methods of controlling chaos: the addition of state feedback control $[12,13]$ and the application of dither signals $[16,17]$.

4.1. State Feedback Control. Cai et al. $[12,13]$ proposed a simple, yet effective, state feedback control algorithm. This method for the $n$-dimensional dynamical system is explained briefly as follows:

$$
\dot{\mathbf{x}}=\mathbf{f}(\mathbf{x}, t),
$$

where $\mathbf{x}(t) \in R^{n}$ is the state vector and $\mathbf{f}=\left(f_{1}, \ldots, f_{i}, \ldots, f_{n}\right)$, where $f_{i}$ is a linear or a nonlinear function and $\mathbf{f}$ includes at least one nonlinear function. Suppose that $f_{k}(x, t)$ is the key nonlinear function leading to chaotic motion in system (13). We add to the equation only one term of state feedback of an available system variable $x_{m}$ that includes $f_{k}(x, t)$, as follows:

$$
\dot{x}_{k}=f_{k}(\mathbf{x}, t)+G x_{m}, \quad k, m \in\{1,2, \ldots, n\},
$$

where $G$ is feedback gain. Other functions retain their original forms. We applied this method to (1a)-(1d) to more clearly elucidate the simple control scheme.
In the absence of state feedback control, (1a)-(1d) exhibits chaotic behavior under the following parameters: $A_{0}=3.5 \mathrm{~V}$ and $\Omega=18.2 \mathrm{~Hz}$. Consider how the addition of state feedback control to the right-hand side of the (1a)-(1d) would affect the results. Figure 7 presents the resulting bifurcation diagram for $\Omega=18.2 \mathrm{~Hz}$ with state feedback control. When $G=0$, (1a)-(1d) displays chaotic motion (see Figure 5). This figure shows that chaotic motion occurs at approximately $G>-7.0$ and disappears at approximately $G \leq-7.0$. When the feedback gain $G$ falls below -7.0 , the system described by (1a)-(1d) exhibits stable periodic motion. After 10 seconds, the control signal is applied to the system to control chaotic oscillation (Figure 8). In so doing, the state feedback control can be used to suppress chaotic motion and improve the performance of a magnetic bearing system with closed-loop control.

4.2. Dither Control. In this section, we describe how chaotic motion can be controlled by injecting an external dither signal to adjust nonlinear terms. A dither is a high-frequency signal introduced to modify the behaviors of nonlinear systems through the elimination of nonlinearities. The ability of a dither signal to average out nonlinearities can be attributed to its high frequency and periodic nature. Dither smoothing techniques were proposed in references $[16,17]$ to stabilize chaotic systems. Some of the most common dither signals were proposed by Cook [21].

(i) Square-wave dither: the simplest type of dither signal is the square-wave signal as shown in Figure 9, where 


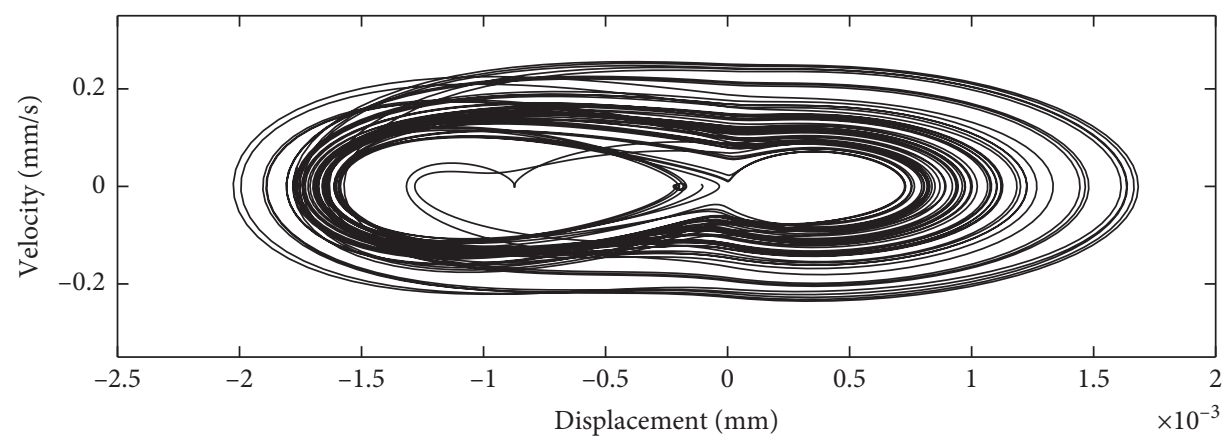

(a)

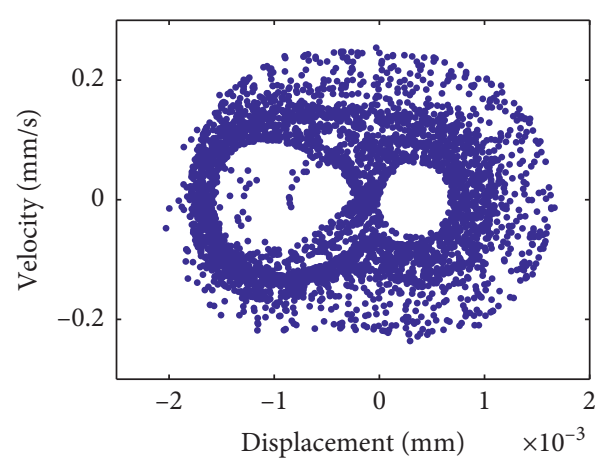

(b)

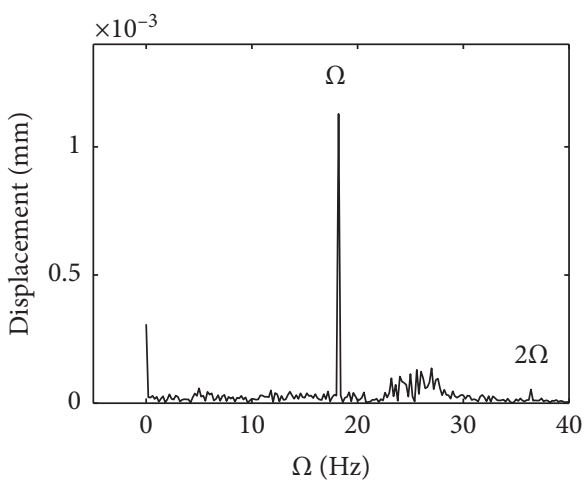

(c)

Figure 5: Chaotic motion for $\Omega=18.2 \mathrm{~Hz}$ : (a) phase portrait; (b) Poincaré map; (c) frequency spectrum.

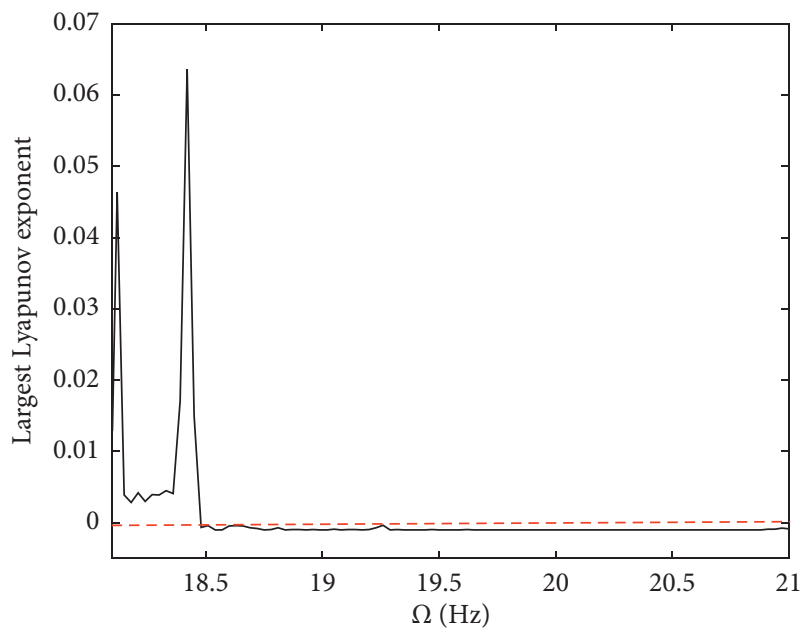

FIgURE 6: Evolutions of the largest Lyapunov exponent.

dither signal takes the constant values $W$ and $-W$ alternately, each holding for a half-period $T / 2$, with $T$ being much smaller than the time constant of the system. The amplitude $W$ is applied in front of the nonlinearity $f(\cdot)$. Thus, the effective value of $\bar{n}$ (the output of the nonlinear element) can be written as [16]

$$
\bar{n}=\frac{1}{2}[f(y+W)+f(y-W)] .
$$

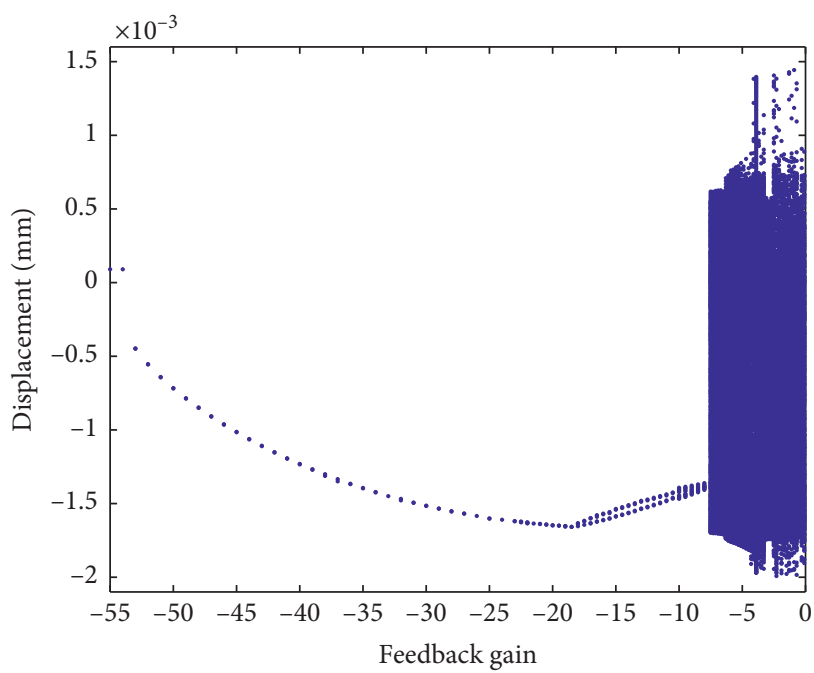

FIgURE 7: Bifurcation diagram of system with state feedback control, where $G$ denotes the feedback gain.

Thus, the system equations can be written as follows:

$$
\dot{y}=\bar{n}
$$

Consider the effect of adding square-wave dither control to the system described in (1a)-(1d), where $\Omega=18.2 \mathrm{~Hz}$. Increasing the amplitude of the squarewave dither signal from $W=0$ to $0.0005 \mathrm{~V}$ would 


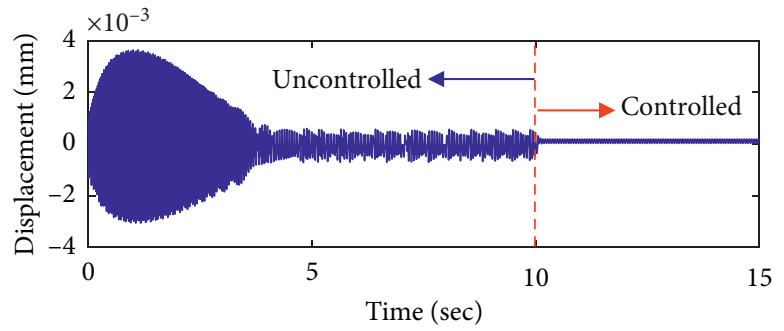

(a)

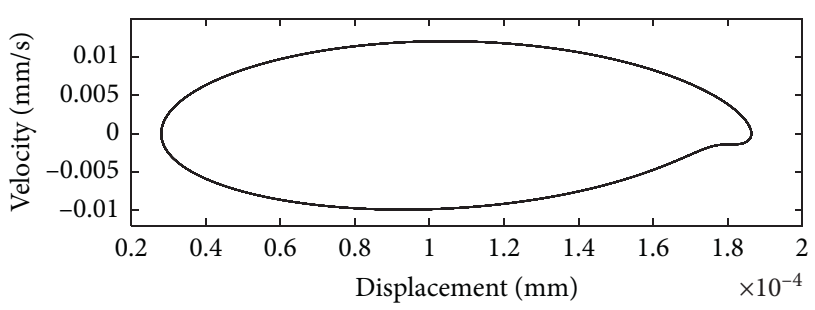

(b)

Figure 8: Transforming chaotic motion to a desired period-one orbit at $G=-50$ and $\Omega=18.2 \mathrm{~Hz}$ where the state feedback control signal is introduced after $10 \mathrm{~s}$ : (a) time responses; (b) phase portrait of the controlled system.

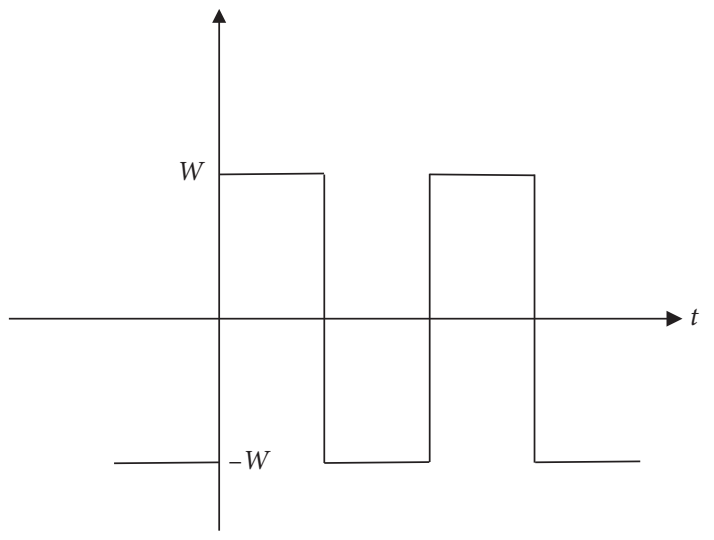

Figure 9: A square-wave dither signal.

change the chaotic behavior to period-one motion. The resulting bifurcation diagram under $\Omega=18.2 \mathrm{~Hz}$ with a square-wave dither signal is shown in Figure 10. Figure 11 shows the effects 10 seconds after applying a control signal to the system in order to illustrate the effectiveness of this controller in controlling chaotic oscillations.

(ii) Sinusoidal dither: one simple dither signal is a highfrequency sinusoid. Here, the effective value of $n$ is its average over a complete period of the sinusoidal dither signal oscillation:

$$
n=\frac{1}{2 \pi} \int_{0}^{2 \pi} f(x+W \sin \theta) \mathrm{d} \theta .
$$

Adding a sinusoidal dither signal to (1a)-(1d) yields the following coupled system:

$$
\begin{aligned}
\dot{\tilde{x}}_{1} & =\tilde{x}_{2}, \\
\dot{\tilde{x}}_{2} & =-d_{1} \tilde{x}_{2}-d_{2} \tilde{x}_{1}+d_{3} \tilde{x}_{3}+d_{4} \widetilde{x}_{4}+n, \\
L \dot{\tilde{x}}_{3}+R \widetilde{x}_{3} & =K_{A}\left(\widetilde{e}_{c 1}+A_{0} \sin \Omega t\right), \\
L \dot{\tilde{x}}_{4}+R \widetilde{x}_{4} & =K_{A}\left(\widetilde{e}_{c 2}+A_{0} \sin \Omega t\right),
\end{aligned}
$$

where

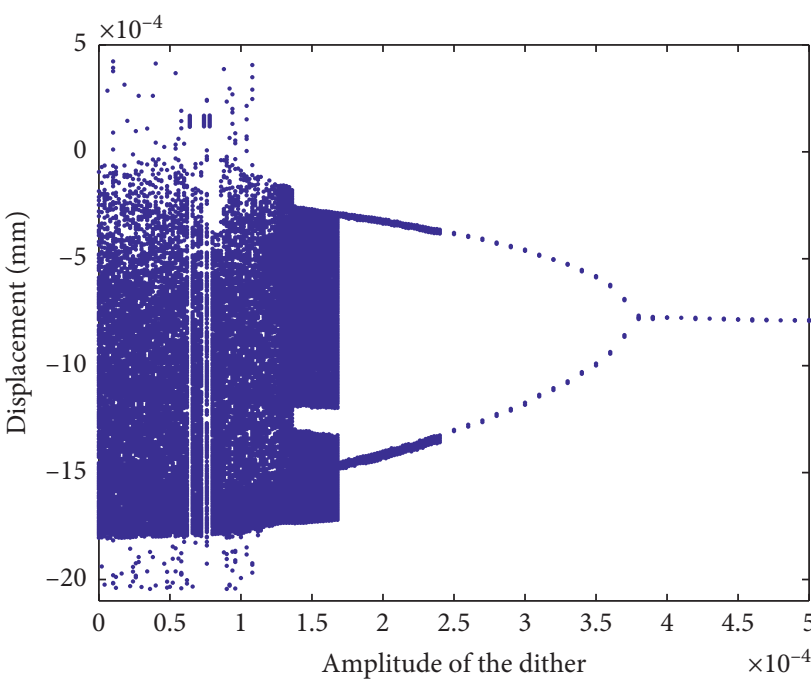

FIGURE 10: Bifurcation diagram of the system with a square-wave dither, where $W$ denotes the amplitude of the dither signal.

$$
\begin{aligned}
\tilde{e}_{c 1}= & K_{H}\left(K_{P} \widetilde{x}_{1}+K_{D} \widetilde{x}_{2}+K_{I} \int \widetilde{x}_{1} \mathrm{~d} t\right), \\
\tilde{e}_{c 2}= & K_{H}\left(-K_{P} \widetilde{x}_{1}-K_{D} \widetilde{x}_{2}-K_{I} \int \widetilde{x}_{1} \mathrm{~d} t\right), \\
n= & \frac{1}{2 \pi} \int_{0}^{2 \pi}\left[d_{5}\left(\widetilde{x}_{1}+W \sin \theta\right)^{2}+d_{6}\left(\widetilde{x}_{1}+W \sin \theta\right)\right. \\
& \cdot\left(\widetilde{x}_{3}+W \sin \theta\right)+d_{7}\left(\widetilde{x}_{1}+W \sin \theta\right)\left(\widetilde{x}_{4}+W \sin \theta\right) \\
& \left.+d_{8}\left(\widetilde{x}_{3}+W \sin \theta\right)^{2}+d_{9}\left(\widetilde{x}_{4}+W \sin \theta\right)^{2}+d_{10}\left(\widetilde{x}_{1}+W \sin \theta\right)^{3}\right],
\end{aligned}
$$

with the following limitations:

$$
\begin{array}{r}
-3.5 \text { volts }<\widetilde{e}_{c 1}<6.5 \text { volts, } \\
-3.5 \text { volts }<\widetilde{e}_{c 2}<6.5 \text { volts, } \\
0.0 \text { amp }<\widetilde{x}_{3} \text { and } \widetilde{x}_{4}<2.0 \text { amp. }
\end{array}
$$

The dither frequency must substantially exceed that of any other frequency used to operate the system in order to ensure that the dither signal does not introduce other undesirable oscillations at the same frequency as the dither signal. For example, Figure 12 presents the resulting 


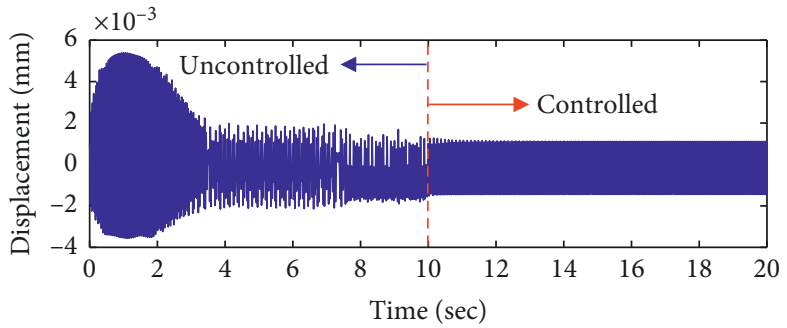

(a)

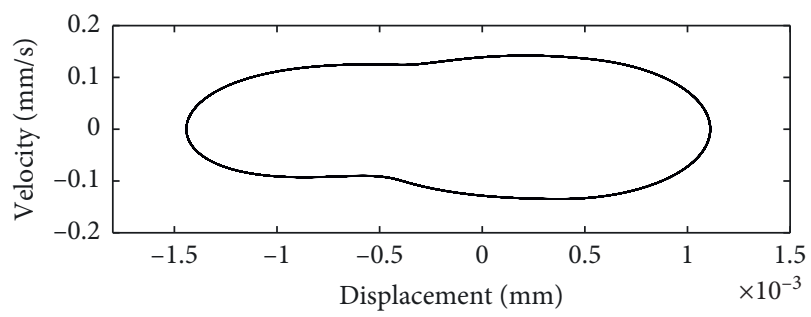

(b)

FIGURE 11: Injecting a square-wave dither signal control which is used to control chaotic motion of the system for $A_{0}=3.5 \mathrm{~V}$ at $\Omega=18.2 \mathrm{~Hz}$. The dither signal ( $W=0.0004)$ is added after 10 seconds: (a) displacement time series; (b) controlled orbit.

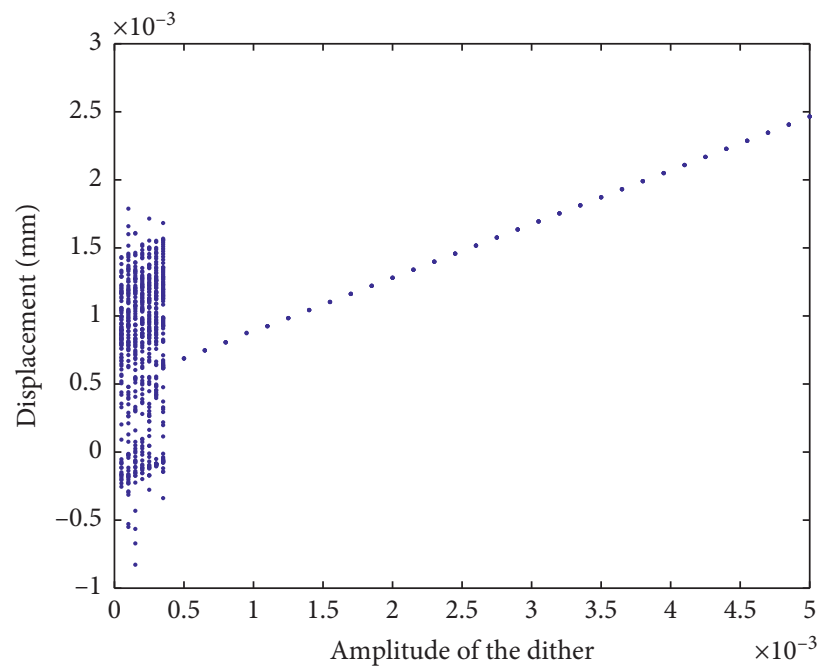

FIGURE 12: Bifurcation diagram of system with a sinusoidal dither, where $W$ is the amplitude of the dither.

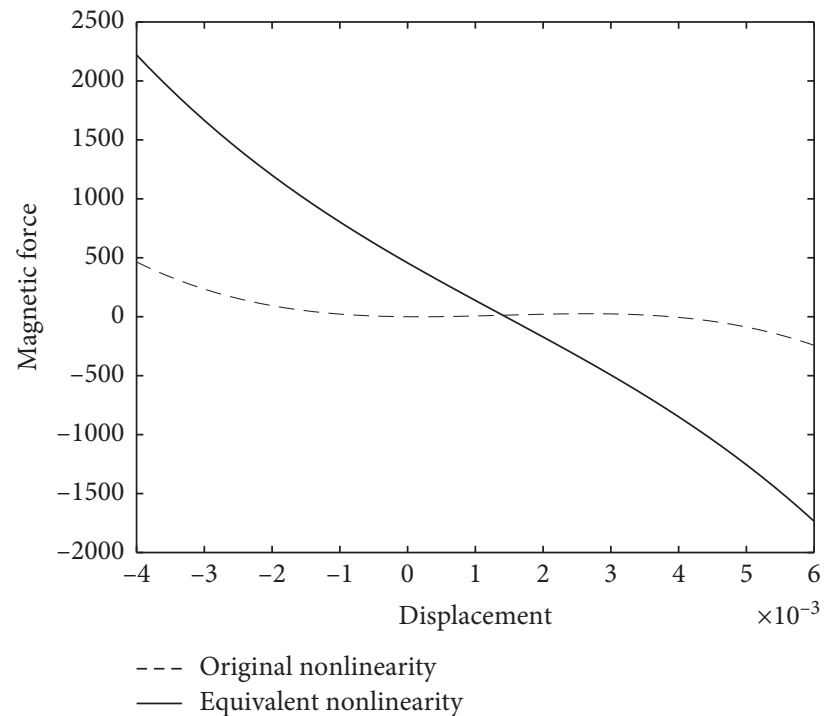

FIGURE 13: Equivalent nonlinearity $n$ (solid line) described by equation (17). Original nonlinearity $f$ (dashed line) is given in equation (1b). 


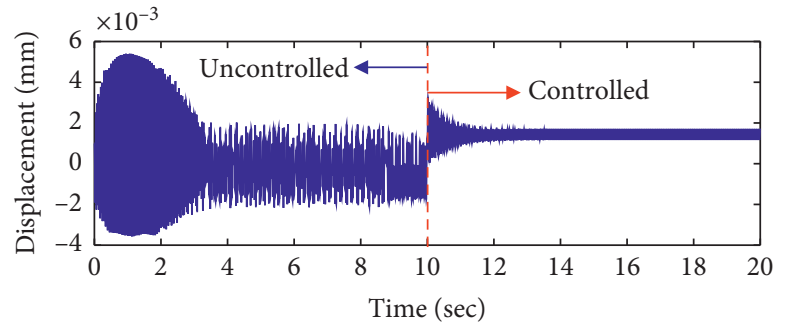

(a)

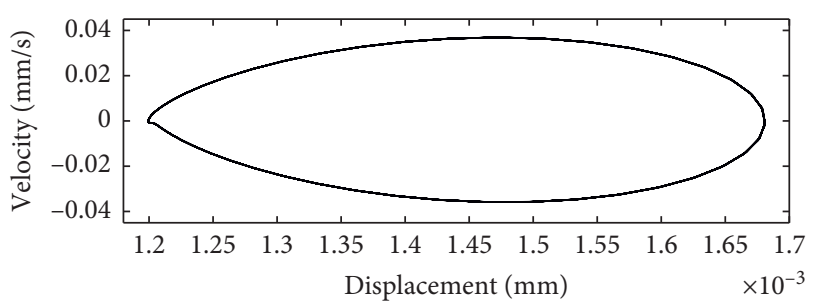

(b)

FIGURE 14: Controlling chaotic motion to desired period-one orbit for $W=0.003 \mathrm{~V}$ and $\Omega=18.2 \mathrm{~Hz}$ : (a) time responses of displacement. The sinusoidal dither signal is injected after $1 \mathrm{~s}$. (b) phase portrait of controlled system.

bifurcation diagram for a system with a sinusoidal dither in which parameter $\Omega$ is $18.2 \mathrm{~Hz}$ and the frequency of the sinusoidal dither is $6000 \mathrm{rad} / \mathrm{s}$. This reveals that a sinusoidal dither with amplitude exceeding $0.0005 \mathrm{~V}$ can convert chaotic behavior into stable periodic motion in a magnetic bearing system. The next steps are setting $W=0.008 \mathrm{~V}$ and plotting the effective nonlinearity $n$ and original nonlinearity $f$, as shown in Figure 13. The time response of displacement 10 seconds after the injection of a sinusoidal dither signal is shown in Figure 14(a). The chaotic behavior is converted into period-one motion. Figure 14(b) presents a phase portrait of the controlled system. Note that the behavior of the system is initially chaotic, but gradually becomes periodic following dither injection.

\section{Conclusions}

This work uses the identified nonlinear model examine global bifurcation and chaos control in the magnetic bearing system. Dynamic behavior over the entire range of parameter values is observable in the resulting bifurcation diagram, which reveals that the magnetic bearing system exhibits period-doubling bifurcations and chaotic motions. The largest Lyapunov exponent derived using the properties of synchronization provided the most powerful tool to measure and analyze chaotic motion in such a system. Controlling chaotic motion is an effective way to prevent chatter vibration in magnetic bearing systems. State feedback control is a simple, yet effective, approach to suppressing chaos. It can be implemented by adding feedback associated with a suitable variable into the original system with control gain sufficient to overcome the development of chaos in dynamic systems prone to chaotic behavior. It is also possible to convert chaotic behavior into a periodic orbit through the injection of a dither signal in front of nonlinearities in a chaotic magnetic bearing system.

Our analysis revealed that the proposed nonlinear model is able to predict the occurrence of bifurcation and chaos in an active magnetic bearing system, which means that it is potentially applicable to a wide range of functions in the design of active magnetic bearing systems. Chaotic behavior must be accepted in some situations; however, it is normally deemed undesirable, as it degrades performance and restricts the operating range of electric and mechanical devices. Accordingly, we applied state feedback and dither control methods to quench chaos, improve the performance of the magnetic bearing system, and prevent the occurrence of chaos behaviors. Furthering the development of magnetically levitated vehicles requires an understanding of their nonlinear dynamic characteristics from the viewpoint of stability, safety, and ride quality at high speeds. Our contribution of this study is that studying nonlinear dynamics and controlling chaotic vibrations in the active magnetic bearing systems will help to advance the development of magnetic transportation systems.

\section{Data Availability}

No data were used to support this study. We only used computer for simulation. Therefore, we can only provide simulation programming, which can be obtained from the corresponding author upon request.

\section{Conflicts of Interest}

The author declares that there are no conflicts of interest regarding the publication of this paper.

\section{Acknowledgments}

This research was supported by the Ministry of Science and Technology in Taiwan, Republic of China, under project number MOST 108-2221-E-212 -010 -MY3.

\section{References}

[1] S. C. Chang and P. C. Tung, "Nonlinear identification of a magnetic bearing system with closed loop control," JSME International Journal Series C Mechanical Systems, Machine Elements and Manufacturing, vol. 42, no. 4, pp. 982-990, 1999.

[2] I. Shimada and T. Nagashima, "A numerical approach to ergodic problem of dissipative dynamical systems," Progress of Theoretical Physics, vol. 61, no. 6, pp. 1605-1616, 1979.

[3] A. Wolf, J. B. Swift, H. L. Swinney, and J. A. Vastano, "Determining Lyapunov exponents from a time series," Physica D: Nonlinear Phenomena, vol. 16, no. 3, pp. 285-317, 1985.

[4] G. Benettin, L. Galgani, A. Giorgilli, and J. M. Strelcyn, "Lyapunov characteristic exponents for smooth dynamical systems and for Hamiltonian systems; a method for computing all of them. Part 1: theory," Meccanica, vol. 15, no. 1, pp. 9-20, 1980. 
[5] G. Benettin, L. Galgani, A. Giorgilli, and J. M. Strelcyn, "Lyapunov characteristic exponents for smooth dynamical systems and for Hamiltonian systems; a method for computing all of them. Part 2: numerical application," Meccanica, vol. 15 , no. 1 , pp. 21-30, 1980.

[6] P. C. Müller, "Calculation of Lyapunov exponents for dynamical systems with discontinuities," Chaos, Solitons \& Fractals, vol. 5, no. 9, pp. 1671-1681, 1995.

[7] N. Hinrichs, M. Oestreich, and K. Popp, "Dynamics of oscillators with impact and friction," Chaos, Solitons \& Fractals, vol. 8, no. 4, pp. 535-558, 1997.

[8] A. Stefanski, "Estimation of the largest Lyapunov exponent in systems with impacts," Chaos, Solitons \& Fractals, vol. 11, no. 15 , pp. $2443-2451,2000$.

[9] E. Ott, C. Grebogi, and J. A. Yorke, "Controlling chaos," Physical Review Letters, vol. 64, no. 11, pp. 1196-1199, 1990.

[10] Z. Shen and J. Li, "Chaos control for a unified chaotic system using output feedback controllers," Mathematics and Computers in Simulation, vol. 132, pp. 208-219, 2017.

[11] S. M. A. Pahnehkolaei, A. Alfi, and J. A. Tenreiro Machado, "Chaos suppression in fractional systems using adaptive fractional state feedback control," Chaos, Solitons \& Fractals, vol. 103, pp. 488-503, 2017.

[12] C. Cai, Z. Xu, W. Xu, and B. Feng, "Notch filter feedback control in a class of chaotic systems," Automatica, vol. 38, no. 4, pp. 695-701, 2002.

[13] C. Cai, Z. Xu, and W. Xu, "Converting chaos into periodic motion by state feedback control," Automatica, vol. 38, no. 11, pp. 1927-1933, 2002.

[14] W. S. Wu, Z. S. Zhao, J. Zhang, and L. K. Sun, "State feedback synchronization control of coronary artery chaos system with interval time-varying delay," Nonlinear Dynamics, vol. 87, no. 3, pp. 1773-1783, 2017.

[15] M. Z. Ullah, F. Mallawi, D. Baleanu, and A. S. Alshomrani, "A new fractional study on the chaotic vibration and statefeedback control of a nonlinear suspension system," Chaos, Solitons \& Fractals, vol. 132, Article ID 109530, 2020.

[16] C. C. Fun and P. C. Tung, "Experimental and analytical study of dither signals in a class of chaotic system," Physics Letters A, vol. 229, no. 4, pp. 228-234, 1997.

[17] Y. M. Liaw and P. C. Tung, "Application of the differential geometric method to control a noisy chaotic system via dither smoothing," Physics Letters A, vol. 239, no. 1-2, pp. 51-58, 1998.

[18] Q. Wei and X. Y. Wang, "Chaos controlling of permanent magnet synchronous motor base on dither signal," Journal of Vibration and Control, vol. 19, no. 16, pp. 2541-2550, 2013.

[19] S. C. Chen and E. T. Mbitu, "Quench limit cycle using different dither signal in a servo motor system," in Proceedings of the International Conference on Advanced Materials for Science and Engineering (ICAMSE), pp. 501-504, Tainan, Taiwan, November 2016.

[20] IMSL, Inc, User's Manual-IMSL MATH/LIBRARY, IMSL, Inc., Louisville, CO, USA, 1989.

[21] P. A. Cook, Nonlinear Dynamical Systems, Prentice-Hall, London, UK, 1994. 